\title{
Decentralised Control Design of Lateral and Vertical Vehicle Dynamics using Passivity
}

\author{
Carlos Villegas $^{* 1}$, Robert Shorten ${ }^{1}$, Martin Corless ${ }^{1,2}$, Mark Readman ${ }^{1}$, and Selim Solmaz ${ }^{1}$ \\ ${ }^{1}$ Hamilton Institute, National University of Ireland-Maynooth, Co. Kildare, Ireland \\ 2 School of Aeronautics \& Astronautics, Purdue University-West Lafayette, IN, USA
}

\begin{abstract}
In this paper, we present a novel methodology to design block-diagonal stable decentralised controllers based on the KalmanYakubovich-Popov lemma. The approach yields closed-loop systems that are robust with respect to parameter uncertainty and structural perturbations. We demonstrate the methodology by designing controllers for the lateral and vertical subsystems of a vehicle emulator; namely, a test vehicle that can mimic the behaviour of a wide range of vehicles.
\end{abstract}

Copyright line will be provided by the publisher

\section{Introduction}

Many situations arise in engineering practice where the principal objective of the control engineer is to integrate a number of control systems that have been designed independently of each other, but which nevertheless interact with each other when implemented in the real plant. This approach is preferred over a centralised controller by reasons such as simplicity and ease of implementation. The automotive industry provides a good example of this strategy where different suppliers are usually responsible for different subsystems. Our objective in this paper is to give a method for controller integration that is robust to certain types of parameter uncertainty, and in particular, to sensor failure. We present a method for designing closed loop systems that are Hurwitz stable, and which have a block diagonal quadratic Lyapunov function. The existence of such a function guarantees the stability of the closed loop system; the individual component systems (ignoring interactions); and guarantees preservation of stability under sensor failure. Our design procedure, which is based on manipulating certain MIMO transfer functions (as opposed to LMI's), allows uncertainty to be expressed in terms of frequency domain arguments, and can be used to design both state and output feedback controllers. The efficacy of our methodology is demonstrated by means of a vehicle emulation control task developed in collaboration with a leading automotive manufacturer.[1]

\section{Passivity-based Design Procedure}

A linear time invariant system described by $\dot{x}=A x$ where

$$
A=\left[\begin{array}{ll}
A_{11} & A_{12} \\
A_{21} & A_{22}
\end{array}\right]
$$

is said to be block-diagonal stable if there exists a positive-definite symmetric $P=\operatorname{diag}\left(P_{11}, P_{22}\right)$ (partitioned in accordance with partition of $A$ ) such that $P A+A^{T} P<0$. Our objective is to design closed-loop systems that are blockdiagonal stable. We use the following theorem.

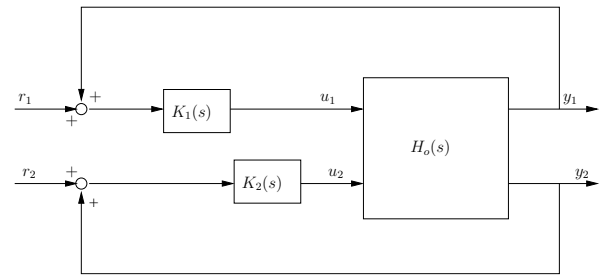

Fig. 1 Decentralised control system.

Theorem 2.1 [2] The following statements are equivalent for a matrix A with structure given in (1).

(i) The system $\dot{x}=$ Ax is block-diagonal stable.

(ii) There exists a matrix $P_{22}=P_{22}^{T}>0$ such that $-P_{22} H$ is strictly positive real where $H(s)=A_{22}+A_{21}\left(s I-A_{11}\right)^{-1} A_{12}$.

(iii) There exists a constant matrix $D$ such that $\left\|D G D^{-1}\right\|_{\infty}<1$ where $G=(H+I)(H-I)^{-1}$.

Consider the feedback system depicted in Fig. 1. Our task is to select $K_{1}, K_{2}$ such that the closed-loop is block diagonal stable with respect to some specified state partition. We present an iterative scheme to design $K_{1}$ and/or $K_{2}$ based on 2.1(iii).

\section{Design methodology.}

Now we describe a practical design procedure analogous to the well known DK-iteration used in $\mu$-synthesis[3].

\footnotetext{
* Corresponding author: e-mail: carlos@villegasramos.net, Phone: +00353 1708 4536, Fax: +00 35317086269
} 
1. Select an initial $D$ such as $D=I$.

2. For a given $D$, find the $K_{1}$ that minimises $\left\|D G D^{-1}\right\|_{\infty}$.

3. For a given $K_{1}$, find a single $D$-scaling matrix that minimises $\left\|D G D^{-1}\right\|_{\infty}$.

4. Repeat steps 2 and 3 until $\gamma:=\left\|D G D^{-1}\right\|_{\infty}$ is minimised for the resulting $D$ and $K_{1}$.

The system is block-diagonal stable if $\gamma<1$. This methodology provides a framework for the design of a block-diagonal stable closed-loop of two interacting subsystems that is robust with respect to parameter uncertainty and structural perturbations. Finding $K_{2}(s)$ can be done by simply rearranging the subsystems.

Comment 1:The proposed design procedure is readily applied to modular design. Often $K_{1}$ is fixed and our task is to find a $K_{2}$ that gives a block-diagonal stable closed-loop subject to the fixed $K_{1}$.

Comment 2:Block-diagonal stabilising state feedback controllers can be obtained using Linear Matrix Inequalities (LMIs). However, it is hard to design output feedback controllers with LMIs. Moreover, the current approach provides a frequency domain framework where one can accommodate frequency domain uncertainty specifications in the system description.

\section{Application to vehicle emulation}

We now apply our design methodology to the design of a decentralised controller for a passenger vehicle equipped with 4wheel-steering and active suspension. Our specific task is to stabilise the lateral and vertical dynamics of a vehicle where $K_{1}$ is to stabilise the lateral dynamics, and $K_{2}$ the vertical dynamics; and to use the resulting controllers to track a set of reference dynamics. While both subsystems clearly affect one another, the requirement for a decentralised structure is a stringent one and comes directly from the automotive manufacturers. A controller for each subsystem was sequentially designed: first the vertical subsystem and then the lateral dynamics at different operating speeds; this latter subsystem is speed-dependent. The simulation results for a mid-sized vehicle emulating a light commercial vehicle are presented in Fig. 2 . In this figure, the reference dynamics (solid) are being tracked by the vehicle outputs (dashed) for the selected J-turn maneuver at $80 \mathrm{~km} / \mathrm{h}$. The motions of the vehicle emulator in passive mode - with no active systems - driven by the same steering maneuver are also plotted for comparison (dotted lines).
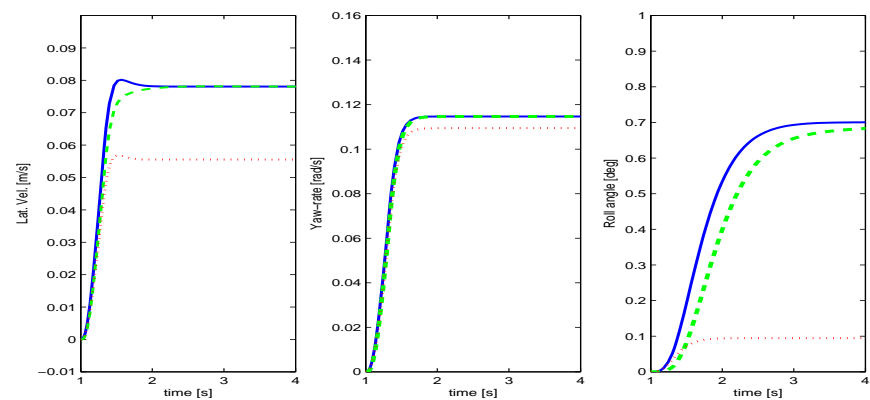

Fig. 2 Emulation results for a light commercial vehicle.

\section{Conclusion}

In this paper, we provide a link between stabilising decentralised controllers and robust control theory based on the KalmanYakubovich-Popov lemma. On this grounds, we sketch a sequential design procedure that profits from the well established $H_{\infty}$ and $\mu$-synthesis design methods. Such methodology provides a framework for the design of decentralised control systems that are robust with respect to parameter uncertainty and structural perturbations. We demonstrate the effectiveness of the procedure with numerical results by applying it to the vehicle emulation problem.

Acknowledgements This work was jointly supported by the EU FP6 Project CEmACS 004175 and the SFI Investigator Award 04/IN1/I478.

\section{References}

[1] C. Villegas, M. Readman, M. Akar, and R. Shorten, D21:Integrated Chassis Control, CEmACS Project Final Report, July 2007.

[2] Carlos Villegas, Decentralised Control of the Lateral and Vertical Dynamics of Passenger Vehicles, in preparation, Hamilton Institute.

[3] Kemin Zhou and John C. Doyle, Essentials of Robust Control (Prentice Hall, 1998). 